\title{
JETS AND CHARGED HADRONS IN HEAVY-ION COLLISIONS WITH THE ATLAS DETECTOR***
}

\begin{abstract}
Helena SAntos
on behalf of the ATLAS Collaboration

LIP — Laboratório de Instrumentação e Física Experimental de Partículas Lisboa, Portugal

(Received October 2, 2017)

Ultrarelativistic heavy-ion collisions at the LHC produce the quarkgluon plasma. Jets are a useful probe to study this state of matter since they are produced at the early stages of the collisions and are expected to be modified as propagating through the medium. One observable is the energy loss lowering the jet yields at a given transverse momentum. Other observables are the modification of the dijet momentum balance and the modification of fragmentation functions. A phenomenon strictly correlated to the jet energy loss is the modification of the charged-hadron momentum spectrum. The large new $\mathrm{Pb}+\mathrm{Pb}$ data sample collected by ATLAS in Run 2 allows for precision measurements of these observables in a wide transverse momentum range and in different centrality and rapidity intervals.
\end{abstract}

DOI:10.5506/APhysPolBSupp.10.1191

\section{Introduction}

A wide research program is ongoing at the Large Hadron Collider with the aim of studying the properties of QCD matter at extreme temperatures and densities. Jets produced in ultra-relativistic heavy-ion collisions constitute a golden probe of such a state of matter. The hard scattered quarks and gluons emerged from these collisions evolve as parton showers that propagate through the hot and dense medium. Constituents of the parton showers emit medium-induced gluon radiation and, as a consequence, the resulting jet loses energy, a phenomenon commonly termed as "jet quenching" [1]. Jets produced in heavy-ion collisions are thus expected to be suppressed at

* Presented at "Excited QCD 2017", Sintra, Lisbon, Portugal, May 7-13, 2017.

** This work was supported in part by FCT under the contract Project IF/01586/ 2014/CP1248/CT0003. 
a given $p_{\mathrm{T}}$, relatively to a sample produced in $p p$ collisions. Their internal structure is also expected to be modified. The large acceptance and high granularity of the ATLAS detector [2] is well-suited to study these phenomena. Results shown in this conference used data produced in $\mathrm{Pb}+\mathrm{Pb}$ and $p p$ collisions at the center-of-mass energies of $2.76 \mathrm{TeV}$ and $5.02 \mathrm{TeV}$. The centrality of the $\mathrm{Pb}+\mathrm{Pb}$ collisions is estimated using the total transverse energy deposited in the forward calorimeters (FCal, $3.2 \leq|\eta|<4.9$ ) and compared to a Glauber Monte Carlo model [3], convoluted with $p p$ data taken at the same beam energy. The $\sum E_{\mathrm{T}}^{\mathrm{FCal}}$ distribution is then divided into percentiles of the total inelastic cross section for $\mathrm{Pb}+\mathrm{Pb}$ collisions. Jets are reconstructed using calorimeter "towers" as input signals to the anti- $k_{\mathrm{T}}$ algorithm, with jet radius parameter size $R=0.4$. The underlying event is estimated and subtracted event-by-event in each calorimeter layer and strip of pseudorapidity after excluding regions subtending jet candidates and corrected for flow modulation.

\section{Results}

The nuclear modification factor $R_{A A}$, defined as the ratio of normalized yields in $\mathrm{Pb}+\mathrm{Pb}$ and $p p$ collision systems, is used to compare the inclusive transverse momentum distributions measured in the two collision systems

$$
R_{A A} \equiv \frac{\left(1 / N_{\text {evt }}\right) \mathrm{d}^{2} N_{\text {jet }}^{\mathrm{PbPb}} /\left.\mathrm{d} p_{\mathrm{T}} \mathrm{d} y\right|_{\text {cent }}}{\left\langle T_{A A}\right\rangle \mathrm{d}^{2} \sigma_{\text {jet }}^{p p} / \mathrm{d} p_{\mathrm{T}} \mathrm{d} y},
$$

where $\left\langle T_{A A}\right\rangle$ stands for the geometric enhancement of per-collision nucleonnucleon luminosity and $N_{\text {evt }}$ is the total number of $\mathrm{Pb}+\mathrm{Pb}$ collisions within a chosen centrality interval. The jet yields, unfolded for detector resolution, bin-to-bin migration and reconstruction inefficiency are shown in Fig. 1. Jets are suppressed increasingly with collisions centrality, reaching a factor of two in central collisions (0-10\%), while showing little dependence on jet transverse momentum and rapidity [4]. These measurements confirm the expectations on the reduction of the jet yields at a given transverse energy due to the interaction of partons in the quark-gluon plasma [1].

A complementary observable to jet production is the measurement of charged particle spectra, as these contribute to the understanding of jet energy loss mechanisms. Tracks are reconstructed using the ATLAS inner detector system in the pseudorapidity range $|\eta|<2.5$ and over the full azimuthal acceptance. Corrections to measured $p_{\mathrm{T}}$ spectra such as fake tracks, bin-to-bin migration, and reconstruction inefficiency are estimated from Monte Carlo simulations. The charged hadron $R_{A A}$ as a function of $p_{\mathrm{T}}$ is shown in Fig. 2. A characteristic $p_{\mathrm{T}}$ shape, which becomes more pronounced with increasing centrality, is observed. The $R_{A A}$ distributions first 


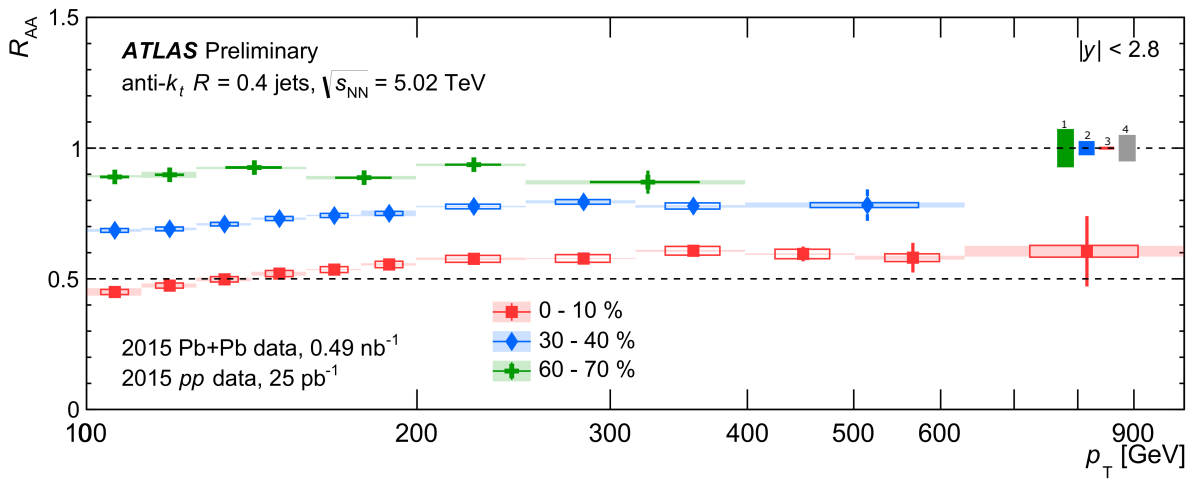

Fig. 1. (Colour on-line) The $R_{A A}$ as a function of jet $p_{\mathrm{T}}$ for jets with $|y|<2.8$ for three centrality bins. The error bars represent statistical uncertainties, the shaded boxes around the data points represent correlated systematic uncertainties, open boxes represent uncorrelated systematic uncertainties. The coloured shaded boxes $(1,2,3)$ at unity represent $\left\langle T_{A A}\right\rangle$ uncertainties (associated to $60-70 \%, 30-40 \%$, and 0-10\% centrality bins, respectively) and the grey shaded box (4) represents the uncertainty on $p p$ luminosity. The horizontal width on the shaded boxes represents the width of the $p_{\mathrm{T}}$ interval and the horizontal widths on the open boxes are arbitrary for better visibility [4].

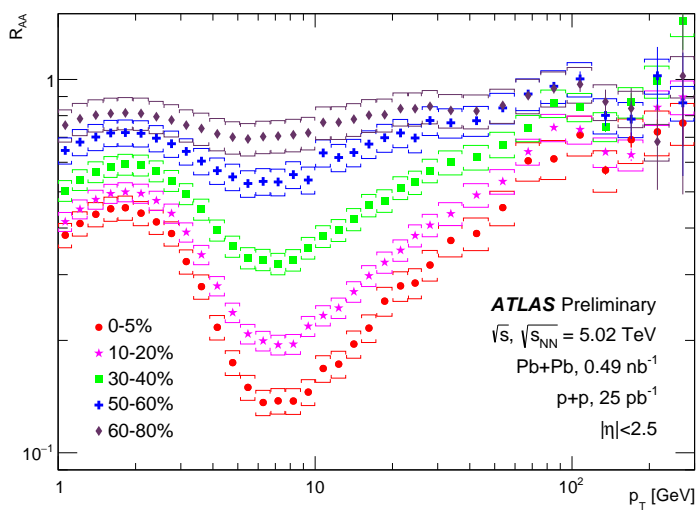

Fig. 2. Nuclear modification factor $R_{A A}$ as a function of $p_{\mathrm{T}}$ for charged hadrons measured in $\mathrm{Pb}+\mathrm{Pb}$ collisions at $\sqrt{s_{N N}}=5.02 \mathrm{TeV}$ for five centrality intervals: $0-5 \%, 10-20 \%, 30-40 \%, 50-60 \%$ and $60-80 \%$. Bars represent the statistical uncertainties and brackets represent the systematic uncertainties [6].

increase reaching a local maximum at $p_{\mathrm{T}}<2 \mathrm{GeV}$, a feature commonly associated with the Cronin effect [5], i.e. hardening of the $p_{\mathrm{T}}$ spectrum in heavy-ion collisions relative to $p p$ collisions, often understood to be due to multiple scattering of partons. At higher $p_{\mathrm{T}}$, the $R_{A A}$ decreases reaching 
a minimum at $p_{\mathrm{T}}=7 \mathrm{GeV}$. The rate of charged particles is noticeably suppressed even in the 60-80\% centrality interval but the suppression is strongest in the most central $0-5 \%$ collisions. Above $7 \mathrm{GeV}$, the $R_{A A}$ generally increase with increasing $p_{\mathrm{T}}$ up to $60 \mathrm{GeV}$, after which the slope changes. Details of this analysis can be found in [6].

Jet fragmentation functions are measured aiming at deeper understanding of the jet energy loss nature and constraining jet quenching models. The jet structure is probed in the $p_{\mathrm{T}}$ range from 100 to $398 \mathrm{GeV}$, using tracks with $p_{\mathrm{T}}>1 \mathrm{GeV}$. The fragmentation functions, defined as $D\left[z\left(p_{\mathrm{T}}\right)\right]=$ $\left(1 / N_{\text {jet }}\right) \mathrm{d} N_{\mathrm{ch}} / \mathrm{d} z\left(p_{\mathrm{T}}\right)$, are studied as a function of the longitudinal momentum fraction, $z=p_{\mathrm{T}}^{\mathrm{ch}} / p_{\mathrm{T}}^{\mathrm{jet}} \cos \Delta R$, and track $p_{\mathrm{T}}$, where $p_{\mathrm{T}}^{\mathrm{ch}}$ stands for the transverse momentum of a charged particle, and $\Delta R$ is the distance between the charged particle and the jet axis. The $D(z)$ and $D\left(p_{\mathrm{T}}\right)$ distributions are background subtracted, corrected for reconstruction inefficiency and unfolded with a 2D Bayesian method. Figure 3 shows the modification of the fragmentation functions, assessed with $R_{D(z)}=\left.D(z)\right|_{\mathrm{Pb}+\mathrm{Pb}} /\left.D(z)\right|_{p p}$ in different ranges of rapidity. An enhancement at low $z$, up to 0.03 , and at $z$ larger than 0.2 , mainly at mid-rapidity, is observed in central collisions. The range $0.03<z<0.2$ shows a clear depletion. The enhancement of fragment yields at low $z$ is consistent with an interpretation in which the energy lost by partons is transferred predominantly to soft particles [7]. Details on this analysis can be found in [8].

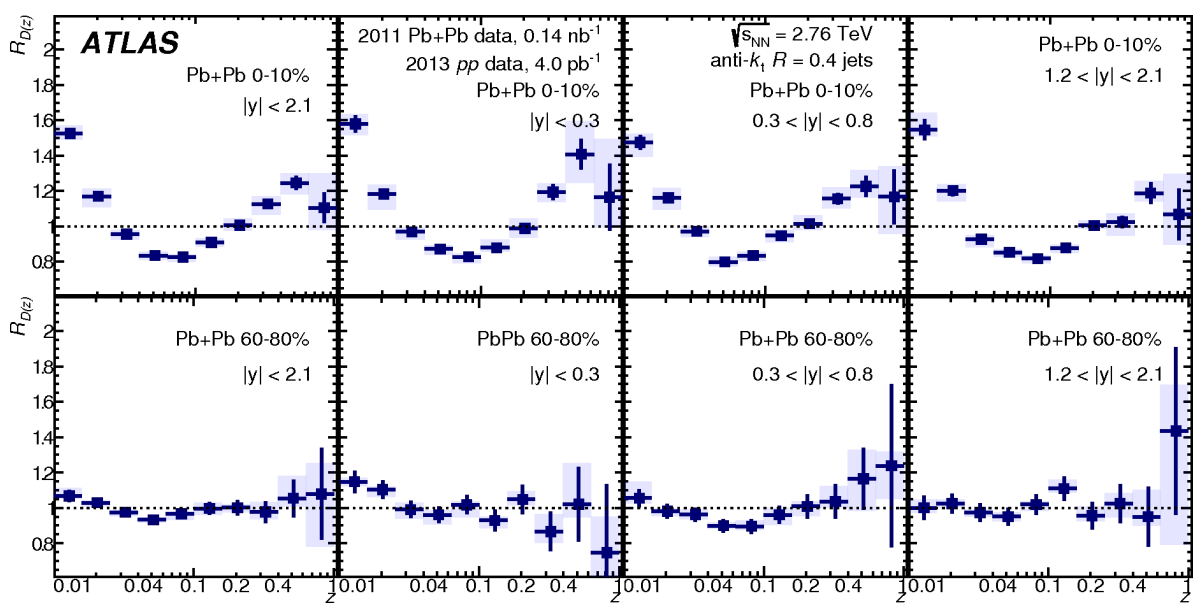

Fig. 3. The ratio $R_{D(z)}$ of unfolded $D(z)$ distributions measured in heavy-ion collisions to unfolded $D(z)$ distributions measured in $p p$ collisions. The $R_{D}(z)$ distributions shown here are evaluated in two different centrality bins (rows) and four different selections in jet rapidity (columns). The error bars on the data points indicate statistical uncertainties, while the shaded bands indicate systematic uncertainties [8]. 
The first indication of jet quenching was given by the observation of large asymmetrical dijet events [9]. Dijets probe differences in the two parton showers since in most of the cases the interaction of the two outgoing partons in the quark-gluon plasma is not identical. A new analysis has been performed using $140 \mu \mathrm{b}^{-1}$ of $\mathrm{Pb}+\mathrm{Pb}$ data taken at $\sqrt{s_{N N}}=2.76 \mathrm{TeV}$ and benefiting from a deeper understanding of the underlying event and analysis conditions. Figure 4 shows the distribution of dijets produced in $\mathrm{Pb}+\mathrm{Pb}$ and $p p$ collisions as a function of $x_{\mathrm{J}}$, the ratio between the transverse momenta of the two leading jets. The measured distributions are unfolded to account for the effects of experimental resolution and inefficiencies on the two-dimensional $\left(p_{\mathrm{T}_{1}}, p_{\mathrm{T}_{2}}\right)$ distributions and then projected into bins of fixed ratio $x_{\mathrm{J}}=p_{\mathrm{T}_{1}} / p_{\mathrm{T}_{2}}$. The observation of large asymmetric dijets in central collisions is striking. The distributions in the two colliding systems
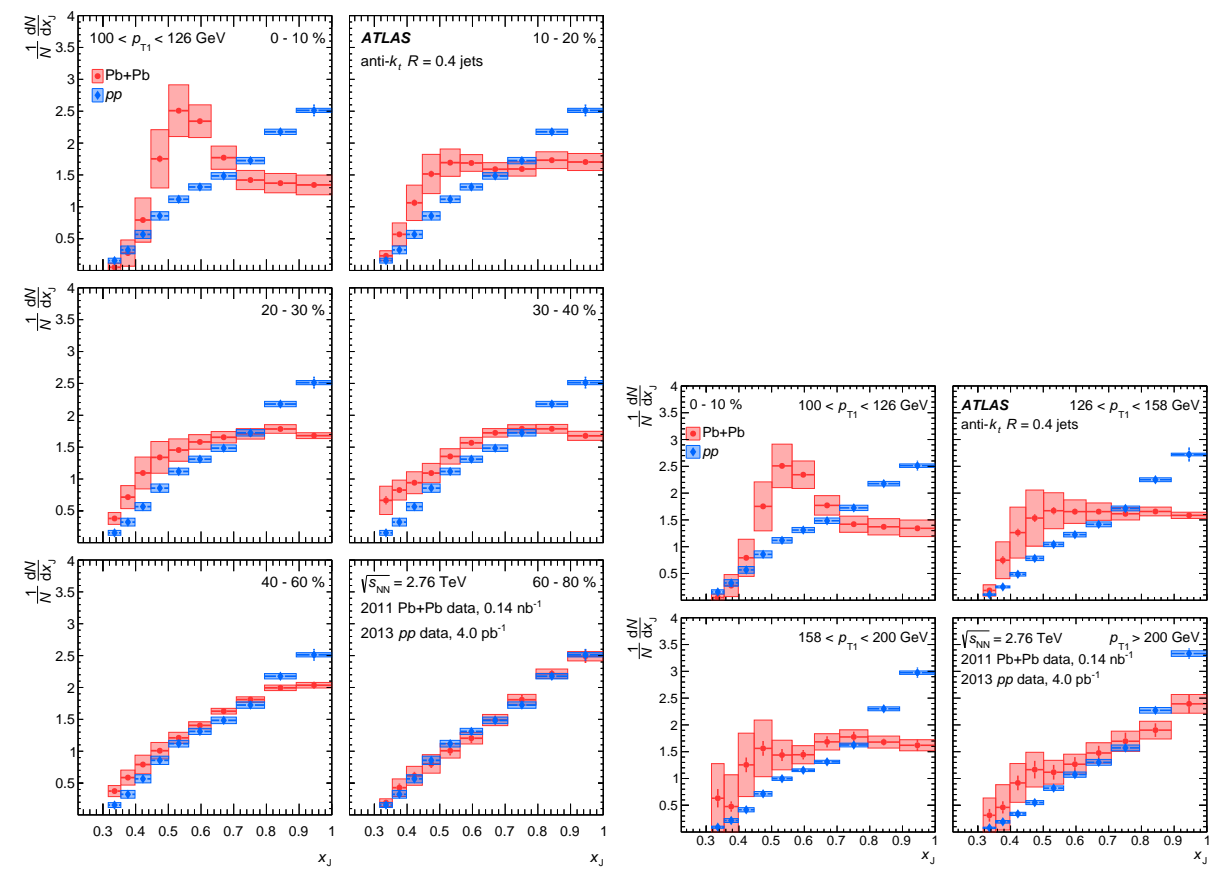

Fig. 4. (Colour on-line) Left: The $(1 / N) \mathrm{d} N / \mathrm{d} x_{\mathrm{J}}$ distributions for jet pairs with $100<p_{\mathrm{T}_{1}}<126 \mathrm{GeV}$ for different collision centralities and for $R=0.4$ jets. The $\mathrm{Pb}+\mathrm{Pb}$ data are shown in grey/red circles, while the $p p$ distribution is shown for comparison in black/blue diamonds and is the same in all panels. Right: The $(1 / N) \mathrm{d} N / \mathrm{d} x_{\mathrm{J}}$ distributions with different selections on $p_{\mathrm{T}_{1}}$, shown for the $0-10 \%$ centrality interval (grey/red circles) and for $p p$ (black/blue diamonds). In both panels the statistical uncertainties are indicated by the error bars, while systematic uncertainties are shown with shaded boxes [10]. 
become increasingly similar with decreasing centrality. Interesting is the study of the variation of the dijet asymmetry with the $p_{\mathrm{T}}$ of the leading jet. A modification in the asymmetry in $\mathrm{Pb}+\mathrm{Pb}$ collisions as the $p_{\mathrm{T}}$ of the leading jet increases is observed, indicating that higher transverse momentum jets lose less energy and so tend to be more balanced. This feature is particularly evident for transverse momentum greater than $200 \mathrm{GeV}$. Details on this analysis can be found in [10].

\section{Conclusions}

Inclusive jets yields are found to be suppressed with collisions centrality, reaching a factor of two in central collisions, while showing little dependence on jet transverse momentum and rapidity. In contrast, the nuclear modification factor of charged hadrons shows a striking non-flat $p_{\mathrm{T}}$ shape which becomes more pronounced with increasing centrality. The jet structure is modified: in central collisions, there is an enhancement of particles at low and high $z$, while a suppression at intermediate $z$ is observed. The $p_{\mathrm{T}}$ balance of dijets produced in $\mathrm{Pb}+\mathrm{Pb}$ collisions is increasingly asymmetric when compared to the $p p$ results as the centrality of the collisions increases. At larger values of $p_{\mathrm{T}_{1}}$, the $x_{\mathrm{J}}$ distributions are observed to narrow and the differences between the distributions in central $\mathrm{Pb}+\mathrm{Pb}$ and $p p$ collisions become much smaller.

\section{REFERENCES}

[1] J.-P. Blaizot, Y. Mehtar-Tani, J. Mod. Phys. E 24, 1530012 (2015) [arXiv: 1503.05958 [hep-ph]] and references therein.

[2] ATLAS Collaboration, JINST 3, S08003 (2008).

[3] M.L. Miller, K. Reygers, S.J. Sanders, P. Steinberg, Annu. Rev. Nucl. Part. Sci. 57, 205 (2007).

[4] ATLAS Collaboration, ATLAS-CONF-2017-009, http://cds.cern.ch/record/2244820

[5] J.W. Cronin et al., Phys. Rev. D 11, 3105 (1975).

[6] ATLAS Collaboration, ATLAS-CONF-2017-012, http://cds.cern.ch/record/2244824

[7] CMS Collaboration, Phys. Rev. C 84, 024906 (2011) [arXiv:1102.1957 [nucl-ex]].

[8] ATLAS Collaboration, Eur. Phys. J. C 77, 379 (2017) [arXiv: 1702.00674 [hep-ex]].

[9] ATLAS Collaboration, Phys. Rev. Lett. 105, 252303 (2010) [arXiv:1011.6182 [hep-ex]].

[10] ATLAS Collaboration, Phys. Lett. B 774, 379 (2017) [arXiv:1706.09363 [hep-ex]]. 